\title{
Manifestações orais decorrentes da radioterapia de cabeça e pescoço
}

\author{
Manifestations or oral manifestations resulting from head and neck radiotherapy \\ Manifestaciones o manifestaciones orales resultantes de la radioterapia de cabeza y cuello
}

Recebido: 12/09/2021 | Revisado: 19/09/2021 | Aceito: 23/09/2021 | Publicado: 25/09/2021

Nívia Castro Binda

ORCID: https://orcid.org/0000-0002-0409-4265

Universidade Federal do Espírito Santo, Brasil

E-mail: nivia_sgp@hotmail.com

Ana Luiza Castro Binda

ORCID: https://orcid.org/0000-0002-6664-2538

Escola São Francisco de Assis, Brasil

E-mail: aninha_sgp@hotmail.com

Sandryelle de Andrade Rodrigues

ORCID: https://orcid.org/0000-0002-0878-3885

Centro Universitário Doutor Leão Sampaio, Brasil

E-mail: sandryellerodrigues24@gmail.com

Leandro Luiz da Silva Loures

ORCID: https://orcid.org/0000-0001-8863-8858

Universidade Federal de Juiz de Fora, Brasil

E-mail: leandrolouresnutri@gmail.com

Anne Caroline Silva Freire de Sá

ORCID: https://orcid.org/0000-0003-3860-1373

Centro Universitário Tabosa de Almeida, Brasil

E-mail: annecarolinefreiree@gmail.com

José Victor Lima Silva

ORCID: https://orcid.org/0000-0002-8539-5456

Centro Universitário Christus, Brasil

E-mail: victorlimasv@hotmail.com

Amanda Gonçalves Franco

ORCID: https://orcid.org/0000-0003-0983-7539 Universidade de Itaúna, Brasil

E-mail: amandagfranco38@gmail.com

Thalita Oliveira da Silva Borba

ORCID: https://orcid.org/0000-0001-5539-7744

Centro Universitário Tabosa de Almeida, Brasil E-mail: thalita.borba00@gmail.com

Pedro Ícaro Bezerra Mariano de Almeida Magalhães

ORCID: https://orcid.org/0000-0001-9953-3015 Faculdade de Medicina Estácio de Juazeiro do Norte, Brasil

E-mail: pedroicaro_bmam@hotmail.com

Edyssa Laryssa da Silva Ferreira de Araújo

ORCID: https://orcid.org/0000-0002-4799-6330

Universidade Federal de Alagoas, Brasil E-mail: edyssa.araujo@foufal.ufal.br

Hana Yasmim Marques Silva de Souza

ORCID: https://orcid.org/0000-0003-0128-7720

Centro Universitário Tiradentes de Pernambuco, Brasil

E-mail: hanayasmim@hotmail.com

Rebeca Luzia Solarte Barbosa

ORCID: https://orcid.org/0000-0003-2021-7584

Centro Universitário do Norte, Brasil

E-mail: solarterebeca@gmail.com

Fernanda da Rosa Savi

ORCID: https://orcid.org/0000-0001-9500-9988

Universidade do Extremo Sul Catarinense, Brasil

E-mail: fefasavi@gmail.com

Jaqueline Lopes Reis

ORCID: https://orcid.org/0000-0003-1685-0336 Universidade de Rio Verde, Brasil

E-mail: jaquelreis@hotmail.com

Matheus Almeida Ramalho

ORCID: https://orcid.org/0000-0002-5607-1313 Universidade Federal de Jataí, Brasil

E-mail: matheusramalho@discente.ufg.br 


\author{
Cleison da Silva Fernandes \\ ORCID: https://orcid.org/0000-0002-8427-699X \\ Centro Universitário UNIFG, Brasil \\ E-mail: ferndscleei@gmail.com \\ Vittor Dorinato de Santana Sátiro \\ ORCID: https://orcid.org/0000-0002-7069-0807 \\ Universidade Paulista, Brasil \\ E-mail: vittorodonto@gmail.com
}

\begin{abstract}
Resumo
O câncer de cabeça e pescoço (CPP) corresponde a tumores localizados na pele, cavidade oral, lábios, glândulas salivares, laringe, faringe, cavidade nasal, seios paranasais, meato acústico externo e ouvido médio. Acomete pessoas de ambos o sexo, porém com maior predileção para o masculino e com idade superior a 40 anos. Os fatores de riscos associados a essa malignidade são tabagismo, etilismo, sobrepeso, exposição a raios ultravioleta, sedentarismo, entre outros. A radioterapia (RT) é o tratamento mais utilizado para CPP, e consiste em diferentes formas de radiações ionizantes que atuam sobre o DNA das células malignas, a fim causar sua morte e/ou impedir sua reprodução. Entretanto, embora seja em menor proporção, devido a maior capacidade de regeneração, a RT afeta tecidos e células saudáveis, ocasionando efeitos adversos que podem afetar o tratamento oncológico e influenciar no bem-estar de saúde do paciente. Diante do exposto, esse estudo objetivou revisar a literatura acerca das manifestações orais decorrentes da radioterapia da cabeça e do pescoço e abordar as estratégias de manejo oral para radioterapia de cabeça e pescoço. Para a construção deste artigo foi feito um levantamento bibliográfico nas bases de dados SciVerse Scopus, Scientific Eletronic Library Online (Scielo), U.S. National Library of Medicine (PUBMED) e ScienceDirect, usando o gerenciador de referências Mendeley. Os artigos foram coletados no período de maio a agosto de 2021 e contemplados entre os anos de 2015 a 2021. A partir da revisão narrativa da literatura, observou-se que as manifestações orais decorrentes da radioterapia de cabeça e pescoço xerostomia, mucosite oral, osteorradionecrose, trismo e disgeusia. Levando em consideração as grandes incidências de CPP, é de extrema importância que os profissionais da saúde estejam familiarizados com as complicações advindas de tratamentos oncológicos.
\end{abstract}

Palavras-chave: Radioterapia; Complicações orais; Câncer de cabeça e pescoço.

\begin{abstract}
Head and neck cancer (CPP) corresponds to assumed tumors in the skin, oral cavity, lips, salivary glands, larynx, pharynx, nasal cavity, paranasal sinuses, external auditory canal and middle ear. It affects people of both sexes, but with greater predilection for males and over 40 years of age. The risk factors associated with this malignancy are smoking, alcohol consumption, overweight, exposure to ultraviolet rays, sedentary lifestyle, among others. Radiotherapy (RT) is the most used treatment for CPP, and consists of different forms of ionizing radiation that act on the DNA of malignant cells in order to cause their death and/or prevent their reproduction. However, although it is in a smaller proportion, due to the greater capacity for regeneration, an RT affects healthy tissues and cells, causing adverse effects that can affect cancer treatment and lead to the patient's health well-being. Given the above, this study aimed to review the literature on oral manifestations resulting from head and neck radiotherapy and address oral management strategies for head and neck radiotherapy. For the construction of this article, a bibliographic survey was carried out in the SciVerse Scopus, Scientific Electronic Library Online (Scielo), National Library of Medicine of the United States (PUBMED) and ScienceDirect databases, using the Mendeley reference manager. The articles were collected from May to August 2021 and covered between 2015 and 2021. From the narrative review of the literature, it was observed that the oral manifestations resulting from radiotherapy of the head and neck xerostomia, oral mucositis, osteoradionecrosis , trismus and dysgeusia. Taking into account the high incidence of PPC, it is extremely important that health professionals are familiarized with the complications arising from cancer treatments.
\end{abstract}

Keywords: Radiotherapy; Oral complications; Head and neck cancer.

\title{
Resumen
}

El cáncer de cabeza y cuello (CPP) corresponde a supuestos tumores en la piel, cavidad oral, labios, glándulas salivales, laringe, faringe, cavidad nasal, senos paranasales, conducto auditivo externo y oído medio. Afecta a personas de ambos sexos, pero con mayor predilección por los varones y mayores de 40 años. Los factores de riesgo asociados a esta malignidad son el tabaquismo, el consumo de alcohol, el sobrepeso, la exposición a los rayos ultravioleta, el sedentarismo, entre otros. La radioterapia (RT) es el tratamiento más utilizado para la CPP, y consiste en diferentes formas de radiación ionizante que actúan sobre el ADN de las células malignas para provocar su muerte y / o prevenir su reproducción. Sin embargo, aunque en menor proporción, debido a la mayor capacidad de regeneración, un RT afecta a los tejidos y células sanos, provocando efectos adversos que pueden afectar al tratamiento del cáncer y derivar en el bienestar de la salud del paciente. Dado lo anterior, este estudio tuvo como objetivo revisar la literatura sobre las manifestaciones orales resultantes de la radioterapia de cabeza y cuello y abordar las estrategias de manejo oral de la radioterapia de cabeza y cuello. Para la construcción de este artículo se realizó un levantamiento bibliográfico en las bases de datos SciVerse Scopus, Scientific Electronic Library Online (Scielo), National Library of Medicine de Estados Unidos (PUBMED) y ScienceDirect, utilizando el gestor de referencias Mendeley. Los artículos fueron recolectados de 
mayo a agosto de 2021 y cubiertos entre 2015 y 2021. A partir de la revisión narrativa de la literatura, se observó que las manifestaciones orales resultantes de la radioterapia de cabeza y cuello son xerostomía, mucositis oral, osteorradionecrosis, trismo y disgeusia. Teniendo en cuenta la alta incidencia de PPC, es de suma importancia que los profesionales sanitarios estén familiarizados con las complicaciones derivadas de los tratamientos oncológicos.

Palabras clave: Radioterapia; Complicaciones orales; Cáncer de cabeza y cuello.

\section{Introdução}

O câncer de cabeça e pescoço (CPP) corresponde a tumores localizados na pele, cavidade oral, lábios, glândulas salivares, laringe, faringe, cavidade nasal, seios paranasais, meato acústico externo e ouvido médio. Acomete pessoas de ambos os sexos, porém com maior predileção para o masculino e com idade superior a 40 anos. Os fatores de riscos associados a essa malignidade são tabagismo, etilismo, sobrepeso, exposição à raios ultravioletas, sedentarismo, entre outros (Chiu et al., 2021).

O CPP é considerado uma das principais causas de morte por todo o mundo. O Instituto Nacional do Câncer (INCA), em 2020, registrou cerca de 43 mil novos casos de CPP no Brasil. O carcinoma de células escamosas é o tipo histológico de câncer mais frequente na região de cabeça e pescoço, sendo a boca o sítio anatômico de maior ocorrência (Tawfik et al., 2021).

O tratamento das neoplasias depende, de forma geral, do estadiamento da lesão que leva em conta o sítio da lesão, presença de linfonodos afetados e de metástases. Atualmente, as medidas terapêuticas para o CPP podem ser executadas pela cirurgia, radioterapia (RT) ou quimioterapia (QT), podendo estas terapêuticas estarem associadas (Mody et al., 2021).

A radioterapia (RT) é o tratamento mais utilizado para CPP, e consiste em diferentes formas de radiações ionizantes que atuam sobre o DNA das células malignas, a fim causar sua morte e/ou impedir sua reprodução. Entretanto, embora seja em menor proporção, devido a maior capacidade de regeneração, a RT afeta tecidos e células saudáveis, ocasionando efeitos adversos que podem afetar o tratamento oncológico e influenciar no bem-estar de saúde do paciente (Kim et al., 2021).

Diante do exposto, esse estudo objetivou revisar a literatura acerca das manifestações orais decorrentes da radioterapia da cabeça e do pescoço e abordar as estratégias de manejo oral para radioterapia de cabeça e pescoço.

\section{Metodologia}

Refere-se a uma revisão integrativa de literatura, de caráter qualitativa. A revisão de literatura permite a busca aprofundada dentro de diversos autores e referenciais sobre um tema específico, nesse caso, as manifestações orais decorrentes da radioterapia de cabeça e pescoço (Pereira et al., 2018).

A fim de que haja direcionamento na pesquisa, delineou-se como questão norteadora: "quais são as manifestações orais decorrentes da radioterapia na região da cabeça e do pescoço?” e “quais são as estratégias de manejo oral para radioterapia de cabeça e pescoço disponíveis na literatura?"

\subsection{Busca Estratégica}

Para a construção deste artigo foi feito um levantamento bibliográfico nas bases de dados SciVerse Scopus, Scientific Eletronic Library Online (Scielo), U.S. National Library of Medicine (PUBMED) e ScienceDirect, com auxílio do Mendeley. Os artigos foram coletados no período de maio a agosto de 2021 e contemplados entre os anos de 2015 a 2021.

A estratégia de pesquisa desenvolvida para identificar os artigos incluídos e avaliados para este estudo baseou-se nos descritores contidos na lista dos Descritores em Ciência da Saúde (DeCS) e suas combinações no idioma português e inglês: [(radioterapia OR radiotherapy OR câncer OR neoplasma OR neoplasm OR tumor) AND (boca OR mouth OR oral OR mandíbula OR jaw OR maxila OR maxilar OR maxilla OR) AND (manifestações clínicas OR clinical findings OR radiological findings)] 


\subsection{Critérios de inclusão e exclusão}

Considerou-se como critério de inclusão os artigos completos disponíveis na íntegra nas bases de dados citadas, nos idiomas inglês e português e relacionados com o objetivo deste estudo.

Os critérios de exclusão foram artigos incompletos, duplicados, resenhas, estudos in vitro e resumos.

\subsection{Seleção de estudos}

A estratégia de pesquisa baseou-se na leitura dos títulos para encontrar estudos que investigassem a temática da pesquisa. Caso atingisse esse primeiro objetivo, posteriormente, os resumos eram lidos e, persistindo na inclusão, era feita a leitura do artigo completo. Quando havia dúvida sobre a inclusão, o artigo era lido por outro autor e, a decisão de inclusão ou exclusão era tomada em consenso.

\subsection{Coleta de dados}

$\mathrm{Na}$ sequência metodológica foi realizada a busca e leitura na íntegra dos artigos pré-selecionados, os quais foram analisados para inclusão da amostra.

\section{Resultados e discussão}

Com base na revisão de literatura feita nas bases de dados eletrônicas citadas, foram identificados 2795 artigos científicos, dos quais 324 estavam duplicados com dois ou mais índices. Após a leitura e análise do título e resumos dos demais artigos outros 2390 foram excluídos. Assim, 81 artigos foram lidos na integra e, com base nos critérios de inclusão e exclusão, apenas 22 artigos foram selecionados para compor este estudo. O fluxograma com detalhamento de todas as etapas de seleção está na Figura 1.

A partir da revisão narrativa da literatura, observou-se que as manifestações orais decorrentes da radioterapia de cabeça e pescoço são xerostomia, mucosite oral, osteorradionecrose, trismo e disgeusia. 
Figura 1 - Fluxograma de identificação e seleção dos estudos.

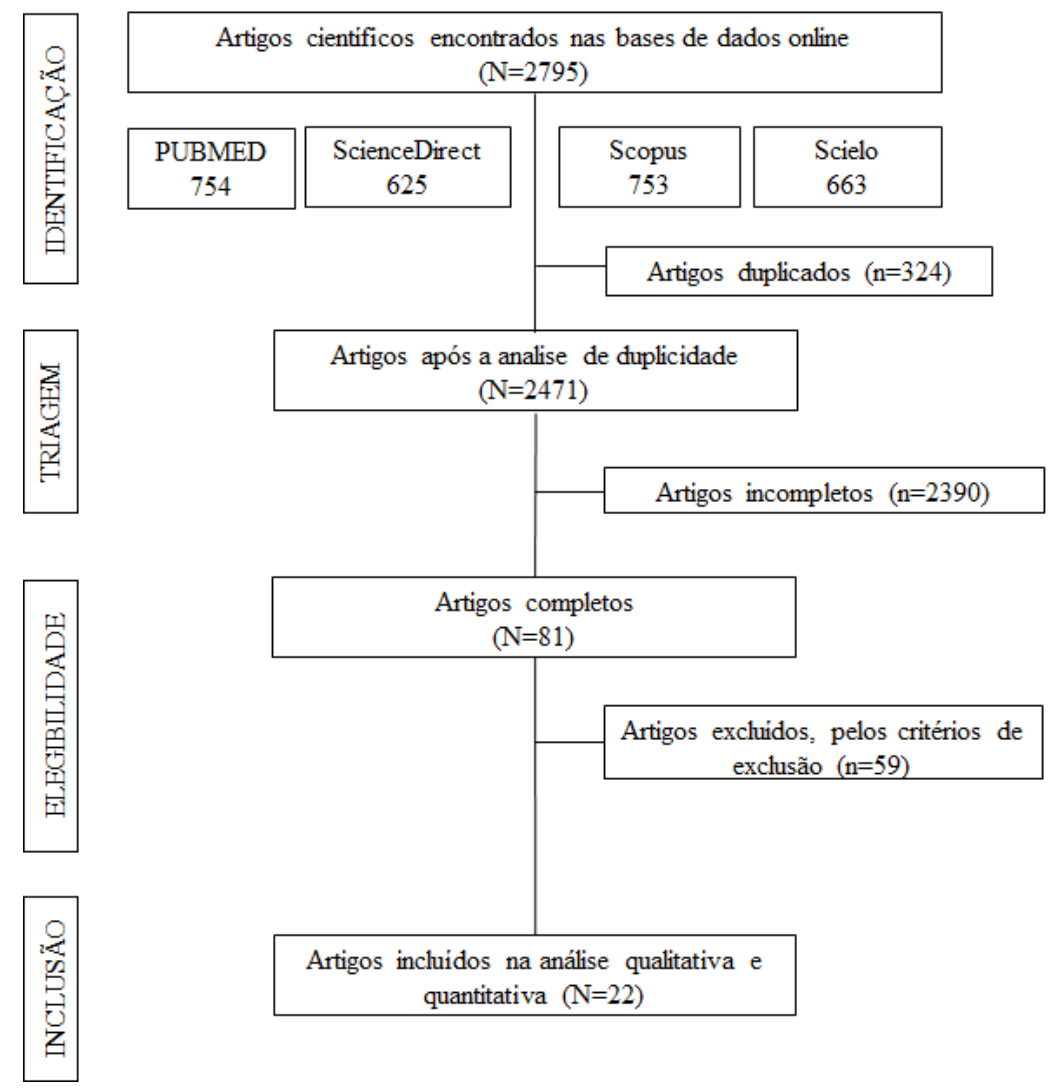

Fonte: Autores (2021).

\subsection{Xerostomia}

A saliva desempenha um papel importante para a limpeza da cavidade bucal devido a sua atividade antimicrobiana. Quando há a alteração do fluxo salivar, os processos fisiológicos encontram-se prejudicados, colaborando para o desenvolvimento da cárie dentária, além de causar desconfortos como dor, dificuldade na mastigação e deglutição (Djordjevic et al., 2016).

O termo xerostomia consiste na sensação subjetiva de boca seca, podendo estar associada, ou não, à hiposalivação. O grau de salivação deve ser medido pelo exame de sialometria, na qual utiliza-se de coletores sobre a saída dos ductos salivares maiores e estimulando-se a salivação, com ácido cítrico. A faixa de 0,4 a 1,5mL/min é considerada normal para o fluxo salivar parotídeo (Weng et al., 2021).

Existem diversos fatores que contribuem com essa condição, incluindo a radioterapia na região de cabeça e pescoço. Isso ocorre, pois, as glândulas salivares apresentam-se sensíveis à radiação, provocando alterações morfológicas e/ou funcionais em sua estrutura, sendo sua gravidade dependente da dose de radiação recebida (Silva et al., 2021).

$\mathrm{O}$ tratamento da xerostomia envolve a orientação sobre a eliminação de agentes que diminuam o fluxo salivar, como o tabaco e o álcool. Além disso, há a possibilidade da utilização de substitutos da saliva e sialogogos, como géis umidificadores, balas sem açúcar e chicletes. Concomitantemente, deve ser feito a aplicação tópica de flúor diariamente, a fim de combater as possíveis doenças cáries causada pela diminuição do fluxo salivar (Djordjevic et al., 2016).

\subsection{Mucosite Oral}

A mucosite oral consiste na inflamação dos tecidos moles da cavidade oral, apresentando sintomatologia dolorosa, ardência e desconforto. Afeta significativamente a qualidade de vida dos pacientes e, em alguns casos, dificulta a continuidade 
do tratamento radioterápico (Soutome et al., 2021). Ocorre na maioria dos pacientes submetidos a RT de cabeça e pescoço, devido a geração de espécies reativas de oxigênio, ocasionando a regulação positiva de citocinas pró-inflamatórias, danificando as células da mucosa e ativando vias moleculares que amplificam a região da lesão na mucosa (Kawashita et al., 2020).

A manifestação consiste, em estágios iniciais da lesão, como uma área de coloração esbranquiçada com ausência de descamação de ceratina. Com a progressão da lesão, há a perda desta camada, com reposição de uma mucosa edemaciada, eritematosa e friável. A evolução da lesão resulta em áreas ulceradas com a presença de membrana superficial fibrinopurulenta amarelada e destacável (Campos et al., 2013). A Organização Mundial da Saúde (OMS) classifica a Mucosite Oral de acordo com seus aspectos clínicos e sintomatologia (Tabela 1).

Tabela 1 - Classificação da Mucosite Oral de acordo com a OMS.

\begin{tabular}{|c|c|c|}
\hline Grau de Mucosite & Exame Clínico & Sintomatologia \\
\hline Grau 0 & Ausente & Ausente \\
\hline Grau 1 & Presença de eritema & Sintomas mínimos \\
\hline Grau 2 & $\begin{array}{l}\text { Áreas eritematosas e } \\
\text { ulceradas }\end{array}$ & $\begin{array}{l}\text { Paciente suporta } \\
\text { alimentação sólida }\end{array}$ \\
\hline Grau 3 & $\begin{array}{l}\text { Áreas eritematosas e } \\
\text { ulceradas }\end{array}$ & $\begin{array}{l}\text { Paciente com } \\
\text { alimentação líquida }\end{array}$ \\
\hline Grau 4 & $\begin{array}{l}\text { Necrose e sangramento } \\
\text { espontâneo }\end{array}$ & $\begin{array}{l}\text { Paciente não consegue } \\
\text { se alimentar por via oral }\end{array}$ \\
\hline
\end{tabular}

Fonte: Autores (2021).

\subsection{Osteorradionecrose}

A osteorradionecrose (ORN) é definida como exposição do tecido ósseo necrótico persistente por mais de três meses em uma área anteriormente exposta a radiação ionizante, acima de 50 Gy (Ribeiro et al., 2018). Acomete, majoritariamente, regiões em que há menor vascularização e maior densidade óssea, sendo, portanto, a mandíbula mais afetada (Frankart et al., 2021).

A ORN é a complicação mais grave da radioterapia de cabeça e pescoço, e, embora sua incidência tenha diminuído com os avanços nas medidas terapêuticas preventivas, ela continua sendo uma complicação mórbida do tratamento oncológico. Consiste na isquemia do osso após intensas radiações e caracteriza-se pela sintomatologia dolorosa, necrose, infecção crônica e, em alguns casos, deformidades permanentes. Em pacientes com lesões avançadas, a ressecção da mandíbula torna-se necessária (Musha et al., 2021).

Histologicamente a lesão apresenta-se com destruição de osteócitos, ausência de osteoblastos e com redução ou ausência da capacidade de formação de novos osteócitos ou osteóide. Também é possível notar endoarterites, hiperemia, hialinização, perda celular, hipovasculação, trombose e fibrose. Radiograficamente, a ORN pode ser interpretada pela presença de áreas radiolúcidas mal definidas, diminuição da densidade óssea com fraturas, destruição da cortical e perda do trabeculado no tecido ósseo esponjoso. Podem estar presentes dor intratável, perfuração da cortical, formação de fístula, ulceração superficial e fratura patológica (Frankart et al., 2021).

A ORN pode ter origem espontânea, ser desencadeada a partir de um trauma ou, ainda, proveniente de focos de infecção, como doença cárie e doença periodontal. Todos os fatores bucais com risco de causar trauma devem ser removidos antes do início da radioterapia. Se necessário, deve-se realizar extrações dentárias antes da RT com, no mínimo, duas semanas de antecedência (Kovarik et al., 2021). 
A Sociedade Alemã de Odontologia e Ciências Oral e Craniomandibular aborda a de sondagem periodontal igual ou superior a $5 \mathrm{~mm}$ e envolvimento de furca como critério para extração de elementos dentários em pacientes que serão submetidos a tratamento com radiação.

\subsection{Trismo}

O trismo é caracterizado pela limitação da abertura da boca afetando diversas atividades diárias como mastigar, engolir, falar e manter a higienização oral. Consequentemente pode levar a desnutrição, perda de peso e dificuldades no tratamento odontológico, colaborando com o aparecimento da doença cárie e periodontal (Scherpenhuizen et al., 2015).

É causado pela contração dos músculos da mastigação, como musculo masseter, temporal e pterigoideos, e da capsula articular, levando o tecido à fibrose. A fisiopatologia da fibrose se inicia pelo processo inflamatório resultante da exposição à radiação, progredindo para uma fase atrófica e fibrótica, normalmente, irreversível (Agarwal et al., 2016).

Atualmente, a radioterapia é realizada concomitantemente a exercícios para controlar a abertura limitada da boca. Há diferentes alongamentos da mandíbula e exercícios de abertura da boca, a fim de aumentar a flexibilidade dos músculos e o movimento das articulações (Shao et al., 2020).

\subsection{Disgeusia}

A disgeusia é definida como um paladar anormal e persistente que afeta pacientes a partir da segunda semana de tratamento com RT e pode se estender por semanas ou meses. A sintomas são percebidos pelo paciente e confirmados por meio de teste convencional do paladar, com uso de sabores padronizados, com base nos sabores básicos, como doce, ácido, salgado e amargo. Juntamente, é possível solicitar a realização da análise elétrica e química dos botões gustativos (Jafari et al., 2021).

As papilas gustativas apresentam-se sensíveis à radiação ionizante, com degeneração de sua estrutura histológica. Estudos mostram que a disgeusia acomete cerca de $70 \%$ dos pacientes submetidos ao tratamento radioterápico, influenciando a redução de apetite e perda de peso (Gómez et al., 2017).

\subsection{Estratégias de manejo oral para radioterapia de cabeça e pescoço}

Kawashita et al. 2014 descreveram uma série de protocolos usados para reduzir os afeitos adversos ocasionados pela radioterapia para tratamento de câncer de cabeça e pescoço. Inicialmente deve ser feito o exame físico oral, a fim de identificar dentes infectados e remove-los. Esta conduta reduz os riscos de osteorradionecrose. Além disso, é necessário manter a cavidade bucal úmida para diminuir os sintomas de xerostomia, candidíase e herpes simples.

O comprometimento das glândulas salivares causando a hipossalivação é um efeito colateral previsível para tratamentos com RT na região da cabeça e do pescoço. Essa condição tem início durante os primeiros estágios do tratamento, progride durante o tratamento e se torna permanente em casos em que a radiação é oferecida acima de $30 \mathrm{~Gy}$. A hipossaliação agrava ainda mais os tecidos inflamados. A pilocarpina é um agente parassimpaticomimético, estimulador de glândulas exócrinas, resultando em salivação, podendo ser utilizada como medida terapêutica durante a RT (Davies \& Thompson, 2015).

A pomada tópica é utilizada como tratamento e prevenção da mucosite oral, a fim de diminuir a sintomatologia dolorosa, reduzir riscos de infecção secundária, favorecer a deglutição e, consequentemente, oferecendo melhor qualidade de vida ao paciente. Deve-se implementar medidas de higiene oral eficaz, incluindo escovação, fio dental e uso de enxagues oral a fim de reduzir os riscos de infeção nessa região (Oladega et al., 2021).

O acompanhamento com o cirurgião dentista deve ser feito em todos os estágios da RT, ao mínimo uma vez na semana, inclusive após o tratamento da radiação. O objetivo do manejo dentário pós tratamento radioterápico incluem a prevenção e tratamento da doença cárie e prevenção da ORN pós exposição da radiação (Kawashita et al., 2020). 
Tabela 2 - Procedimentos orais.

\author{
Pré-Radioterapia \\ Exame Oral \\ Extração de dentes infectados
}

Durante a Radioterapia

Administração de cloridrato de pilocarpina

Cuidados orais profissionais semanais

Enxague oral e substituto da saliva

Utilização de pomada de esteroides à base de azeite

\author{
Pós-Radioterapia \\ Aplicação tópica de flúor \\ Consultas ao Consultório Odontológico
}

Fonte: Kawashita et al. (2014).

\title{
4. Considerações Finais
}

A partir das trajetórias bibliográficas dos pesquisadores, esse estudo abordou as manifestações orais decorrentes da radioterapia de cabeça e do pescoço, abordando seus conceitos, características clínicas e medidas terapêuticas.

Os resultados desta pesquisa revelam cinco principais manifestações na região bucal provenientes da radioterapia da cabeça e do pescoço, sendo elas xerostomia, mucosite oral, osteorradionecrose, trismo e disgeusia. Levando em consideração as grandes incidências de CPP, é de extrema importância que os profissionais da saúde estejam familiarizados com as complicações advindas de tratamentos oncológicos.

Com isso, o levantamento bibliográfico sobre as manifestações orais decorrentes da radioterapia de cabeça e do pescoço se mostra importante, pois envolve estudos retrospectivos que possuem relação com o tema proposto e, ao mesmo, tempo, criase a possibilidade futura de novos pesquisadores abordarem o tema, criando novos trabalhos, para que haja redução dos efeitos adversos do tratamento radioterápico, melhorando o bem-estar do paciente.

\section{Referências}

Agarwal, P., Shiva Kumar, H. R., \& Rai, K. K. (2016). Trismus in oral cancer patients undergoing surgery and radiotherapy. Journal of Oral Biology and Craniofacial Research, 6, S9-S13. https://doi.org/https://doi.org/10.1016/j.jobcr.2016.10.004

Campos, L., Carvalho, D. L. C., Castro, J. R., \& Simões, A. (2013). Laserterapia no tratamento da mucosite oral induzida por quimioterapia: relato de caso. Revista Da Associacao Paulista de Cirurgioes Dentistas, 67(2), 102-106.

Chiu, Y.-H., Tseng, W.-H., Ko, J.-Y., \& Wang, T.-G. (2021). Radiation-induced swallowing dysfunction in patients with head and neck cancer: A literature review. Journal of the Formosan Medical Association. https://doi.org/https://doi.org/10.1016/j.jfma.2021.06.020

Davies, A. N., \& Thompson, J. (2015). Parasympathomimetic drugs for the treatment of salivary gland dysfunction due to radiotherapy. Cochrane Database of Systematic Reviews, 2015(10). https://doi.org/10.1002/14651858.CD003782.pub3

Djordjevic, V., Djokic, G., Domic, D., Zivkovic, N., Jankovic, L., Milicic, B., \& Dejanovic, S. D. (2016). P.3.d.013 - Hyposalivation and xerostomia in schizophrenic patients on psychotropic medications. European Neuropsychopharmacology, 26, S537. https://doi.org/https://doi.org/10.1016/S0924977X(16)31576-0

Frankart, A. J., Frankart, M. J., Cervenka, B., Tang, A. L., Krishnan, D. G., \& Takiar, V. (2021). Osteoradionecrosis: Exposing the Evidence Not the Bone. International Journal of Radiation Oncology*Biology*Physics, 109(5), 1206-1218. https://doi.org/https://doi.org/10.1016/j.ijrobp.2020.12.043

Gómez, G. J. Á., Camacho, R. V. L., Torres, J. E. B., Gómez, S. M. B., Álzate, D. P. C., Ross, P. A. C., \& Arévalo, J. E. H. (2017). Alterations found in the mouth of patients treated with head and neck radiotherapy. Medellin, Colombia. Revista Odontológica Mexicana, 21 (2), e86-e96. https://doi.org/https://doi.org/10.1016/j.rodmex.2017.05.012 
Jafari, A., Alaee, A., \& Ghods, K. (2021). The etiologies and considerations of dysgeusia: A review of literature. Journal of Oral Biosciences. https://doi.org/https://doi.org/10.1016/j.job.2021.08.006

Kawashita, Y., Soutome, S., Umeda, M., \& Saito, T. (2020). Oral management strategies for radiotherapy of head and neck cancer. Japanese Dental Science Review, 56(1), 62-67. https://doi.org/https://doi.org/10.1016/j.jdsr.2020.02.001

Kim, S. Y., Beer, M., \& Tshering Vogel, D. W. (2021). Imaging in head and neck cancers: Update for non-radiologist. Oral Oncology, 120 , 105434. https://doi.org/https://doi.org/10.1016/j.oraloncology.2021.105434

Kovarik, J. P., Voborna, I., Barclay, S., Iqbal, M. S., Cunnell, M., Kelly, C., Willis, N., Kennedy, M., \& Kovarik, J. (2021). Osteoradionecrosis after treatment of head and neck cancer: A comprehensive analysis of risk factors with a particular focus on role of dental extractions. British Journal of Oral and Maxillofacial Surgery. https://doi.org/https://doi.org/10.1016/j.bjoms.2021.03.009

Mody, M. D., Rocco, J. W., Yom, S. S., Haddad, R. I., \& Saba, N. F. (2021). Head and neck cancer. The Lancet. https://doi.org/https://doi.org/10.1016/S01406736(21)01550-6

Musha, A., Shimada, H., Kubo, N., Kawamura, H., Okano, N., Sato, H., Kaminuma, T., Okada, K., Anakura, M., Adachi, A., Shirai, K., Saitoh, J., Yokoo, S., Chikamatsu, K., \& Ohno, T. (2021). Clinical features and dosimetric evaluation of carbon ion radiation-induced osteoradionecrosis of mandible in head and neck tumors. Radiotherapy and Oncology, 161, 205-210. https://doi.org/https://doi.org/10.1016/j.radonc.2021.06.022

Oladega, A., Mojdami, D., Hope, A., Watson, E., \& Glogauer, M. (2021). The process of developing consensus guidelines by Dental Oncologists for preradiotherapy dental care in head and neck cancer patients using the modified Delphi technique. Journal of Evidence Based Dental Practice, 101620. https://doi.org/https://doi.org/10.1016/j.jebdp.2021.101620

Pereira, A., Shitsuka, D., Parreira, F., \& Shitsuka, R. (2018). Método Qualitativo, Quantitativo ou Quali-Quanti. In Metodologia da Pesquisa Científica. https://repositorio.ufsm.br/bitstream/handle/1/15824/Lic_Computacao_Metodologia-Pesquisa-Cientifica.pdf?sequence=1. Acesso em: 28 março 2020.

Ribeiro, G. H., Chrun, E. S., Dutra, K. L., Daniel, F. I., \& Grando, L. J. (2018). Osteonecrosis of the jaws: a review and update in etiology and treatment. Brazilian Journal of Otorhinolaryngology, 84(1), 102-108. https://doi.org/10.1016/j.bjorl.2017.05.008

Scherpenhuizen, A., van Waes, A. M. A., Janssen, L. M., Van Cann, E. M., \& Stegeman, I. (2015). The effect of exercise therapy in head and neck cancer patients in the treatment of radiotherapy-induced trismus: A systematic review. Oral Oncology, 51(8), 745-750. https://doi.org/https://doi.org/10.1016/j.oraloncology.2015.05.001

Shao, C.-H., Chiang, C.-C., \& Huang, T.-W. (2020). Exercise therapy for cancer treatment-induced trismus in patients with head and neck cancer: A systematic review and meta-analysis of randomized controlled trials. Radiotherapy and Oncology, 151, 249-255. https://doi.org/https://doi.org/10.1016/j.radonc.2020.08.024

Silva, I. M. V, Donaduzzi, L. C., Perini, C. C., Couto, S. A. B., Werneck, R. I., de Araújo, M. R., Kurahashi, M., Johann, A. C. B. R., Azevedo-Alanis, L. R., Vieira, A. R., \& Couto-Souza, P. H. (2021). Association of xerostomia and taste alterations of patients receiving antineoplastic chemotherapy: A cause for nutritional concern. Clinical Nutrition ESPEN, 43, 532-535. https://doi.org/https://doi.org/10.1016/j.clnesp.2021.03.006

Soutome, S., Yanamoto, S., Nishii, M., Kojima, Y., Hasegawa, T., Funahara, M., Akashi, M., Saito, T., \& Umeda, M. (2021). Risk factors for severe radiationinduced oral mucositis in patients with oral cancer. Journal of Dental Sciences, 16(4), 1241-1246. https://doi.org/https://doi.org/10.1016/j.jds.2021.01.009

Tawfik, G. M., Mostafa, E. M., Alshareef, A., Hmeda, A. Ben, Khaled, S., Abdelwahed, K. A., Mahran, S. A., Agage, H. S., Amer, A. E., Emara, N. S., ElQushayri, A. E., Ebied, A., \& Huy, N. T. (2021). Association between radiotherapy and obstructive sleep apnea in head and neck cancer patients: A systematic review and meta-analysis. Auris Nasus Larynx, 48(6), 1126-1134. https://doi.org/https://doi.org/10.1016/j.anl.2021.04.014

Weng, C.-T., Huang, S.-L., Yang, H.-W., Kao, C.-C., Wei, C.-C., \& Huang, Y.-F. (2021). Oral microbiota in xerostomia patients - A preliminary study. Journal of Dental Sciences. https://doi.org/https://doi.org/10.1016/j.jds.2021.08.007

Y, K., S, H., M, F., M, U., \& T, S. (2014). Prophylactic bundle for radiation-induced oral mucositis in oral or oropharyngeal cancer patients. Journal of Cancer Research \& Therapy, 2(1), 9-13. https://doi.org/10.14312/2052-4994.2014-2 